\title{
Preface to the Track
}

\section{Conceptual Analysis and Ontological Modelling in Information Systems}

\section{Anwendung der Konzeptanalyse und ontologische Model- lierung in der Wirtschaftsinformatik}

\author{
Heinz Dreher', Torsten Reiners'2, Stefan Voß², Robert Williams ${ }^{3}$ \\ ${ }^{1}$ Curtin Business School, Curtin University, Perth \\ ${ }^{2}$ Institute of Information Systems (IWI), University of Hamburg \\ ${ }^{3}$ Blue Wren Software Pty. Ltd., Perth
}

With Web 2.0, the creation of digital content using all types of media accelerated as a result of increasing Internet accessibility, social communities who share and collect information as well as improved applications allowing everyone to become a producer. The current usage of tags and asynchronous linking provides a means to reveal some basic structural elements within the vast 'repositories of knowledge' and generally in the seemingly endless proliferation of data but ignores the needs of (basic) users with respect to differentiate expert knowledge from 'data' (opinion, unverified reports, and other material disguised amateurish gossip and musings. Users wish to retrieve precise results on questions asked in imprecise natural languages, and to overcome any cultural or language barrier, or to extract additional information such as context, temporal relationships, concept interconnectedness, and alternative media documents representing like content.

The next generation of the Web is called 3.0 and is supposed to be 'intelligent' in that it emphasizes machine-facilitated understanding of information by incorporating, for example, semantic networks, machine learning, autonomous agents, artificial intelligence and distributed databases. Of especial importance is the 'understanding' of content by applications, requiring agents to operate autonomously, and the provision of service oriented architectures where missing components are automatically searched for and integrated and provided via a natural interface to the user.

One popular approach for progressing issues relating to concept 'understanding' being used in recent times is ontology based. Ontologies are an explicit, formal specification of common conceptual classification schemes and generally describe a hierarchy of concepts using a subsumption relation. From a theoretic understanding, ontologies also describe semantic relations between concepts allowing 
the distinguishing of different objects with the same identifier (domain knowledge). Concepts represent the meaning behind 'objects', i.e. to understand and express similarity or differences among objects, provide a classification, and identify objects as belonging. Conceptual analysis is the examination and analysis of texts to identify and mark concepts for 'understanding', detecting the context, or scenario, and other forms of sophisticated information-rich structures.

This track reviews state-of-the-art theories and applications of conceptual analysis in the context of Information Science. The importance of understanding the content rather than manipulating and managing the documents is increasing as the amount of data is no longer manageable by humans, especially considering influences of language, culture and required expert knowledge. The three papers presented in this track explore the semantic aspects of text based documents.

Assisting the Discovery and Reuse of Document-based Knowledge using Semantic Metadata by Hinnerk Brïgmann illustrates the basis for an approach to assist in the enterprise wide lifecycle management of electronic documents by utilizing the context of document access by knowledge workers. From this context data one can deduct semantic relations among documents and business-domain specific entities that can be combined into a semantic network. Querying the resulting network allows for the discovery and reuse of unstructured documents.

Electronic product catalogues are the basis for offering and selling products in online market places. These catalogues have to provide a semantically precise description of product features to allow for effective matchmaking of products and customer requests. At the same time, the description has to follow a common terminology allowing the integration with the catalogues of other providers on the same market place. Pbilipp Nowakowski and Heiner Stuckenscbmidt in their paper Ontology-based Product Catalogues: An Example Implementation argue for the use of ontologies as a basis for defining product catalogues. More precisely, they suggest using an ontological model as a basis for encoding product information as formal class descriptions in the web ontology language OWL.

Emanuel Reiterer, Heinz Dreher, and Christian Gütl present Automatic Concept Retrieval with Rubrico, where the software prototype Rubrico was developed for automated essay grading. For this task concepts are retrieved, which are used to get a summary of the learning subject for the purpose of matching those concepts against a specific essay for use in marking. Rubrico works with electronic textual input in multiple formats such as text, html or pdf. It extracts concepts from these files using several algorithms and provides the option to alter the retrieved concepts (stored as an ontology) through human support.

\section{Acknowledgement}

We greatly appreciate the support that we received from the following members of the program committee of our track: Christian Gütl (Graz, Austria), Felix Mödritscher (Vienna, Austria), Alan Rea (Kalamazoo/Michigan, USA). 